\title{
Synthesis and Aggregation Behavior of N-benzyl-N-hexylacrylamide Terpolymer
}

\author{
Bin $X u^{1, a}$ \\ ${ }^{1}$ Department of Chemical Engineering, Binzhou University, Binzhou, 256603, China; \\ acnxubin@126.com
}

Keywords: Synthesis, N-benzyl-N-hexylacrylamide terpolymer, viscosity, fluorescent probe, DLS

\begin{abstract}
Amphiphilic polymer of N-benzyl-N-hexylacrylamide terpolymer were synthesized using free radical micellar polymerization method. Apparent viscosity, fluorescent probe and DLS was employed to study the aggregation behavior of $\mathrm{P}(\mathrm{AM}-\mathrm{NaA}-\mathrm{BHAM})$. The critical aggregation concentration (CAC) of $\mathrm{P}(\mathrm{AM}-\mathrm{NaA}-\mathrm{BHAM})$ is about $800 \mathrm{mg} / \mathrm{L}$. Above CAC, aggregation formed by intermolecular association of hydrophobic groups can induce large quantities of hydrophobic microdomains. Below CAC, $\mathrm{P}(\mathrm{AM}-\mathrm{NaA}-\mathrm{BHAM})$ in the solution has only one kind of hydrodynamic radius of about $100 \mathrm{~nm}$ which is the hydrodynamic radius of intra-molecular association. Above CAC, there are two kinds of hydrodynamic radius of about $100 \mathrm{~nm}$ and $1000 \mathrm{~nm}$, respectively. The $1000 \mathrm{~nm}$ is the hydrodynamic radius of inter-molecular association. As polymer concentration increased, more and more intra-molecular hydrophobic association turned into inter-molecular association.
\end{abstract}

\section{Introduction}

Amphiphilic polymers contain segments that have a tendency to associate in selective solvents. The segments are usually hydrophobic groups and may be randomly distributed along the chain or they may be grouped in blocks [1]. Aggregation can be formed from both intra and intermolecular interactions and their respective contribution is a function of the polymer concentration [2-4].

Amphiphilic polymers are mostly used for application such as enhanced oil recovery, drug release, etc. At concentrations above $\mathrm{CAC}$, intermolecular hydrophobic associations dominate polymer behavior in solution which can result in an increase in the hydrodynamic size of the polymer as well as the apparent viscosity $[5,6]$. The hydrophobic microdomains formed can dissolve organic compounds as well as crude oil which can enhance the displacement efficiency during EOR process.

In this study, an amphiphilic polymer of N-benzyl-N-hexylacrylamide terpolymer $\mathrm{P}(\mathrm{AM}-\mathrm{NaA}-\mathrm{BHAM})$ was synthesized using free radical micellar polymerization method. Apparent viscosity, fluorescent probe and DLS was employed to study the solution properties of $\mathrm{P}(\mathrm{AM}-\mathrm{NaA}-\mathrm{BHAM})$ solutions to illustrate the aggregation behavior in solutions.

\section{Material and Methods}

\subsection{Materials.}

Acrylamide (AM) was twice recrystallized from acetone, dried under vacuum. Sodium acrylate $(\mathrm{NaA})$ was formed by the reaction of acrylic acid (AA) and sodium hydroxide at the same molar ratio. Sodium dodecyl sulphate (SDS) was twice recrystallized from ethanol. Potassium persulphate (KPS) and sodium bisulfite (SBS) were recrystallized from deionized water. All reagents were obtained from Sinopharm Chemical Reagent Co., Ltd. All aqueous solutions were prepared using deionized water. N-benzyl-N-hexylacrylamide (BHAM) was synthesized by the reaction of N-benzyl-N-hexylamine and acryloyl chloride in ice bath for $12 \mathrm{~h}$. The structures of BHAM and the terpolymer are illustrated in Fig. 1. 


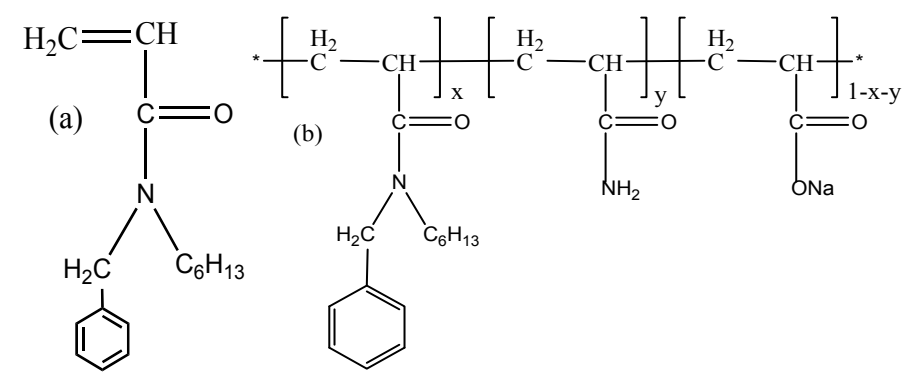

Fig. 1 Structures of BHAM and the terpolymer: (a) BHAM; (b) P(AM-NaA-BHAM)

\subsection{Synthesis of P(AM-NaA-BHAM).}

$\mathrm{P}(\mathrm{AM}-\mathrm{NaA}-\mathrm{BHAM})$ was prepared by using the micellar polymerization technique [5]. The polymerization conditions are: the overall concentration of monomers was $20 \mathrm{wt} \%$, and the concentration of the initiator KPS was $0.5 \mathrm{wt} \%$ relative to the total monomer feed while the molar ration of SBS and KPS is 1:1; the molar ratio of SDS to hydrophobic monomer (SMR) was 25 and the hydrolysis degree and hydrophobic monomer content are $30 \mathrm{~mol} \%$ and $1 \mathrm{~mol} \%$, respectively. The reaction was carried out at $30{ }^{\circ} \mathrm{C}$ for $6 \mathrm{~h}$ with purging nitrogen and vigorous stirring and the product of viscous polymer gel was precipitated into excessive acetone to remove unreacted monomers and surfactant for 3 times and then dried in vacuum at $45{ }^{\circ} \mathrm{C}$ for $48 \mathrm{~h}$.

\subsection{Viscosity Measurements.}

$\mathrm{P}(\mathrm{AM}-\mathrm{NaA}-\mathrm{BHAM})$ solutions were prepared by dissolve polymer in deionized water with gentle stirring at room temperature for $4 \mathrm{~h}$; then the solutions were kept for $12 \mathrm{~h}$ at $45{ }^{\circ} \mathrm{C}$ to remove air bubbles. The apparent viscosity of $\mathrm{P}(\mathrm{AM}-\mathrm{NaA}-\mathrm{BHAM})$ solutions was measured using a NDJ-5S viscometer $\left(6 \mathrm{rpm}, 45^{\circ} \mathrm{C}\right)$.

\subsection{Fluorescence Spectroscopy.}

The fluorescence spectroscopy experiments were performed using a FluoroMax-4 Spectrofluorometer (HORIBA JOBIN YVON Inc.). A pyrene stock solution $\left(1.0 \times 10^{-3} \mathrm{~mol} / \mathrm{L}\right)$ was prepared in methanol and the final pyrene concentration in $\mathrm{P}(\mathrm{AM}-\mathrm{NaA}-\mathrm{BHAM})$ solutions was $1.0 \times 10^{-6} \mathrm{~mol} / \mathrm{L}$. The sample of this solution was gently stirred for $24 \mathrm{~h}$ to ensure the incorporation of the molecular probe into polymer hydrophobic microdomains. All samples were excited at 335 $\mathrm{nm}$ and the emission spectra of pyrene showed vibronic peaks at $\lambda_{1}=372 \mathrm{~nm}$ (intensity $\mathrm{I}_{1}$ ) and $\lambda_{3}$ $=382 \mathrm{~nm}$ (intensity $\mathrm{I}_{3}$ ). The ratio between the fluorescence intensities of peaks $\mathrm{I}_{1}$ and $\mathrm{I}_{3}$ was used to evaluate the polarity of the local microenvironment of pyrene.

\subsection{Dynamic light scattering.}

DLS is used to characterize size of various particles including proteins, polymers, micelles and nanoparticles. For the dynamic light scattering measurements, samples solutions were filtered through $0.1-\mathrm{mm}$ membrane filters before measurement and measured at room tempereture. Data were obtained and analyzed with the Malvern Zetaseizer Nano instrument and AutoPro analysis software. The hydrodynamic radius $\left(\mathrm{R}_{\mathrm{H}}\right)$ is derived according to Stokes-Einstein equation.

\section{Results and Discussion}

The apparent viscosity of $\mathrm{P}(\mathrm{AM}-\mathrm{NaA}-\mathrm{BHAM})$ solutions is shown in Fig. 2. The apparent viscosity of $\mathrm{P}(\mathrm{AM}-\mathrm{NaA}-\mathrm{BHAM})$ solution increased dramatically at polymer concentration of about $800 \mathrm{mg} / \mathrm{L}$. The CAC of $\mathrm{P}(\mathrm{AM}-\mathrm{NaA}-\mathrm{BHAM})$ is about $800 \mathrm{mg} / \mathrm{L}$. The large increase in viscosity is caused by extensive intermolecular association which leads to network structures in $\mathrm{P}(\mathrm{AM}-\mathrm{NaA}-\mathrm{BHAM})$ solutions. 


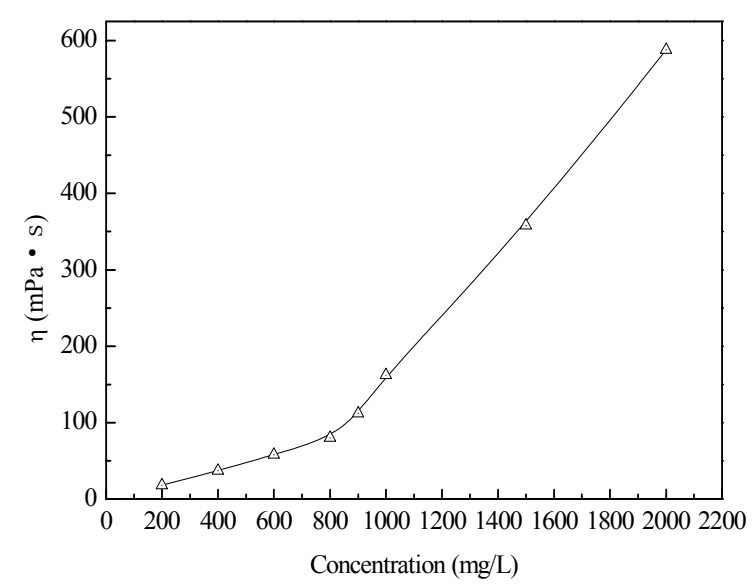

Fig. 2 Apparent viscosity of $\mathrm{P}(\mathrm{AM}-\mathrm{NaA}-\mathrm{BHAM})$ solutions

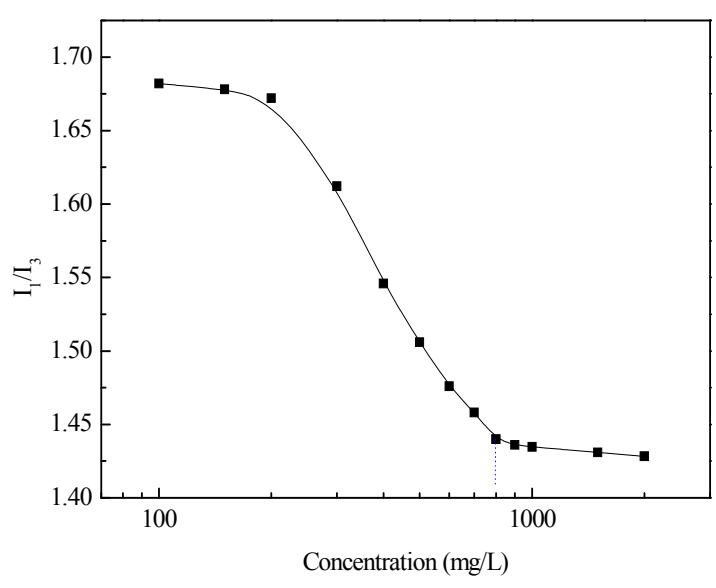

Fig. 3 Variation of $I_{1} / I_{3}$ as a function of polymer concentration

Fig. 3 illustrates the variation of the $\mathrm{I}_{1} / \mathrm{I}_{3}$ ratio with $\mathrm{P}(\mathrm{AM}-\mathrm{NaA}-\mathrm{BHAM})$ concentration. At low polymer concentrations $(<200 \mathrm{mg} / \mathrm{L})$, the $\mathrm{I}_{1} / \mathrm{I}_{3}$ ratio of $\mathrm{P}(\mathrm{AM}-\mathrm{NaA}-\mathrm{BHAM})$ solution was about 1.68 . As polymer concentration increased, a sharp decrease in $\mathrm{I}_{1} / \mathrm{I}_{3}$ was observed, revealing an increasingly apolar local microenvironment of the pyrene in polymer solution. This phenomenon indicates that hydrophobic intramolecular interactions between the hydrophobic groups occurred firstly, which can induce the formation of hydrophobic microdomains to dissolve pyrene. Intermolecular interactions occurred above CAC (about $800 \mathrm{mg} / \mathrm{L}$ ). Above CAC extensive intermolecular association leads to network structures and large quantities of hydrophobic microdomains.

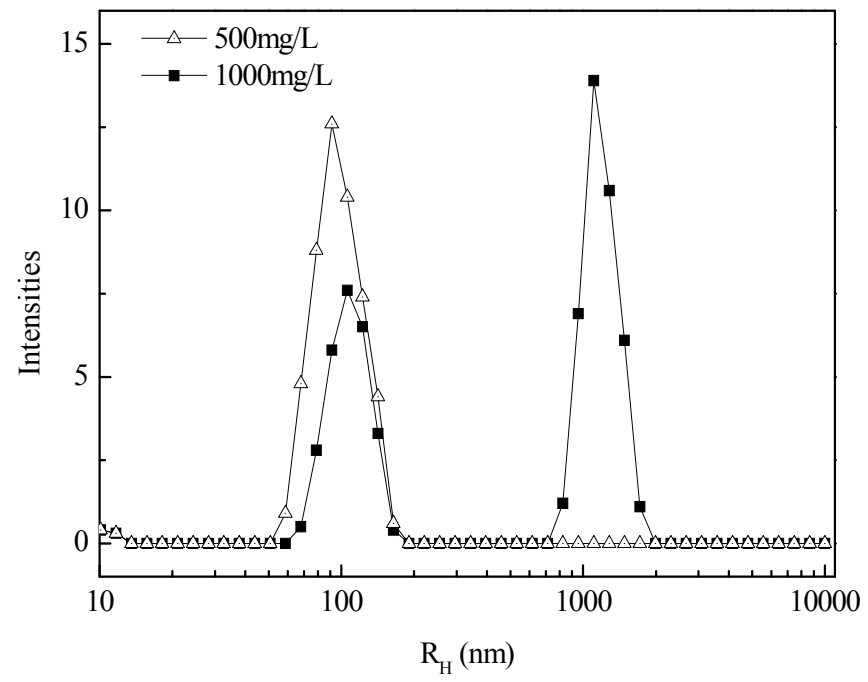

Fig. 4 The apparent hydrodynamic radius distribution curve

Dynamic light scattering (DLS) was utilized to measure the size of polymers in aqueous media. The diameters reported are similar to micelles consisting of polymer chains. Fig.4 shows that, the amphiphilic polymer in the solution below CAC has only one kind of hydrodynamic radius and the median radius is about $100 \mathrm{~nm} .100 \mathrm{~nm}$ is the hydrodynamic radius of intra-molecular hydrophobic association. Above CAC, there are two kinds of hydrodynamic radius of about $100 \mathrm{~nm}$ and $1000 \mathrm{~nm}$, respectively. The $1000 \mathrm{~nm}$ is the hydrodynamic radius of inter-molecular association. As $\mathrm{P}(\mathrm{AM}-\mathrm{NaA}-\mathrm{BHAM})$ concentration increased, more and more intra-molecular hydrophobic association turned into inter-molecular association. 


\section{Conclusions}

Amphiphilic polymer of N, N-dibutyl acrylamide terpolymer were synthesized using free radical micellar polymerization. The critical aggregation concentration (CAC) of $\mathrm{P}(\mathrm{AM}-\mathrm{NaA}-\mathrm{BHAM})$ is about $1000 \mathrm{mg} / \mathrm{L}$. Above CAC, aggregation formed by intermolecular association of hydrophobic groups can induce large quantities of hydrophobic microdomains. Below CAC, P(AM-NaA-BHAM) in the solution has only one kind of hydrodynamic radius of about $100 \mathrm{~nm}$ which is the hydrodynamic radius of intra-molecular association. Above $\mathrm{CAC}$, there are two kinds of hydrodynamic radius of about $100 \mathrm{~nm}$ and $1000 \mathrm{~nm}$, respectively. The $1000 \mathrm{~nm}$ is the hydrodynamic radius of inter-molecular association. As polymer concentration increased, more and more intra-molecular hydrophobic association turned into inter-molecular association.

\section{Acknowledgments}

This research was supported by the Natural Science Foundation of Shandong Province of China (grant Number ZR2015PB007) and the Doctoral Scientific Research Foundation of Binzhou University (2014Y15).

\section{References}

[1]. Chassenieux C, Nicolai T, Benyahia L. Rheology of associative polymer solutions. Curr. Opin. Colloid In., 2011, 16:18-26.

[2]. Volpert E, Selb J, Candau F. Associating behavior of polyacrylamides hydrophobically modified with dihexylacrylamide. Polymer, 1998, 39: 1025-1033.

[3]. Zhao Y, Zhou J, Xu X, et al. Synthesis and characterization of a series of modified polyacrylamide. Colloid and Polymer Science, 2009, 287: 237-241.

[4]. Zhu ZY, Jian O, Paillet S, et al. Hydrophobically modified associating polyacrylamide (HMPAM) synthesized by micellar copolymerization at high monomer concentration. European Polymer Journal, 2007, 43: 824-834.

[5]. Xue W, Hamley IW, Castelletto V, et al. Synthesis and characterization of hydrophobically modified polyacrylamides and some observations on rheological properties. European Polymer Journal, 2004, 40:47-56.

[6]. Volpert E, Selb J, Candau F. Associating behaviour of polyacrylamides hydrophobically modified with dihexylacrylamide. Polymer 1998, 39(5):1025-1033. 